\title{
Action-Potential Broadening and Endogenously Sustained Bursting Are Substrates of Command Ability in a Feeding Neuron of Pleurobranchaea
}

\author{
RHANOR GILLETTE, MARTHA U. GILLETTE, AND WILLIAM J. DAVIS \\ The Thimann Laboratories, University of California, Santa Cruz, California 95064
}

\section{SUMMARY AND CONCLUSIONS}

1. The ventral white cells (VWCs) of the buccal ganglion of Pleurobranchaea, so named for their position and color, are a bilateral pair of neuron somata. Each sends a single axon out its contralateral stomatogastric nerve and has a dendritic field originating close to the soma.

2. The VWCs exhibit spontaneous episodes of prolonged depolarization (duration 1-4 min) accompanied by repetitive actionpotential activity and separated by regular intervals of 3-30 min. Such prolonged burst episodes can be triggered by short pulses of depolarizing current. During the repetitive activity of the spontaneous bursts or that driven by imposed depolarization, the action potentials progressively broaden to 5-16 times their initial duration.

3. During spontaneous bursting or activity driven by imposed depolarization, the cyclic motor output of the feeding network is initiated or accelerated with a latency corresponding with the development of appreciable VWC spike broadening. When broadening of antidromic VWC spikes is suppressed by imposed hyperpolarization of the soma, the frequency of feeding cycles is significantly lower than when broadened spikes are allowed to develop. When trains of spikes are driven by depolarizing current, the motor output of the feeding network is not initiated until the VWC spikes have broadened to a repeatable "threshold" duration, regardless of the intensity of the depolarizing current.

4 . The endogenous production of pro- longed burst episodes, triggered by depolarizing current pulses, and progressive spike broadening can be demonstrated in the surgically isolated VWC soma.

5. The paired VWCs are strongly electrically coupled and display highly synchronous activity. They receive synaptic inputs from many previously identified interneurons of the feeding network and are thus reciprocally coupled within the network.

6. These results demonstrate that the capacity of this neuron to generate broadened action potentials during repetitive activity confers the ability to command coordinated motor-network output. The appropriate repetitive activity can be produced endogenously in the form of prolonged bursts of spikes.

\section{INTRODUCTION}

Feeding behavior in the carnivorous marine gastropod Pleurobranchaea consists of coordinated rhythmic biting and swallowing movements of the feeding apparatus, including the radular and lateral teeth and the muscular esophagus (4). These movements are controlled by a neural network whose elements and interconnections are partially known (e.g., Refs. 5, 8, 10, 17, 18). Among the central elements of the feeding network we have identified several populations of neurons, which, when stimulated, elicit the central motor output pattern underlying feeding $(9,10)$.

This paper describes a newly identified pair of cells, the ventral white cells (VWC), capable of driving feeding output in the 
isolated buccal ganglion. In this paper we demonstrate that the command ability of the VWCs depends on broadening of action potentials consequent to repetitive firing. We demonstrate further that repetitive firing occurs in isolated nervous systems in the form of prolonged, recurrent bursts that are sustained endogenously within the VWC. Forthcoming papers will examine the ionic basis of spike broadening and bursting and the possible roles of cyclic nucleotides and intracellular calcium in modulating these phenomena.

\section{MATERIALS AND METHODS}

\section{Neuron morphology}

The morphology of the VWCs in the buccal ganglion was studied using the intracellular cobalt-staining procedure (14). The neurons were filled with $1 \mathrm{M} \mathrm{CoCl}_{2}$ either intrasomatically by pressure injection $(n=1)$ or backfilled through the axon in the contralateral stomatogastric nerve $(n=3)$. Cobalt filling and subsequent development of the stain and histological procedures were performed as described previously (8). Cleared whole mounts were examined and photographed in the light microscope. Reconstruction of neuron morphology was done by tracing photographs and adding finer details from direct microscopic observation. The axon path was confirmed by electrophysiological mapping (described below), and similar methods were used to study the branching of the axon over the esophagus.

\section{Recording methods}

Multiple extracellular recordings were made using suction electrodes on the cut stumps of nerve roots and connectives or en passant from intact nerves. Switching circuits allowed electrical stimulation through the suction electrodes. Single or dual intracellular recordings were made with $3 \mathrm{M} \mathrm{KCl}$-filled glass microelectrodes and displayed and photographed on a storage oscilloscope or recorded on a Brush-Gould eightchannel chart recorder. The response capability of the chart-recorder pens $(100 \mathrm{~Hz}$ for $1 \mathrm{~cm}$ and $40 \mathrm{~Hz}$ for full-scale 4-cm sine waves) caused slight clipping of the amplitude of the fastest action potentials, but comparison with photographic records showed that it was adequate for reproducing the waveforms of prolonged spikes, their undershoots, and synaptic potentials. Movements of the buccal mass in the semiintact preparation (see below) were registered by a force transducer attached by a small hook to the muscle of the radular sac.

\section{Types of preparation}

After dissection from the animal, ganglia were pinned to Sylgard in a cooled water-jacketed preparation dish. Temperature was maintained at $12-14^{\circ} \mathrm{C}$. Ganglia were bathed in either filtered seawater, the filtered blood of the donor animal, or an artificial saline $(\mathrm{mM})\left(420 \mathrm{NaCl}, 25 \mathrm{MgCl}_{2}, 25\right.$ $\mathrm{MgSO}_{4}, 10 \mathrm{KCl}, 10 \mathrm{CaCl}_{2}$, and 5 Tris- $\mathrm{HCl}$ buffer, $\mathrm{pH} 7.5$ ). In some experiments the connective tissue was softened to facilitate electrode penetration by a 6 - to 12 -minute incubation in $0.2 \%$ pronase (grade B, Calbiochem) in seawater at room temperature. Three types of dissected preparation were used: the isolated buccal ganglion, the isolated brain and buccal ganglia connected by the cerebrobuccal connectives, and the semi-intact preparation. The semi-intact preparation included the entire buccal mass with a 5$\mathrm{cm}$ length of esophagus and the attached buccal ganglion and brain. The buccal mass was pinned loosely to Sylgard so as to allow the rostrocaudal movements of feeding to occur, while the buccal ganglion was stabilized on a small micromanipulated platform above the buccal mass for intracellular stimulation and recording of VWC activity. The outer, muscular connective tissue sheath overlying the ventral side of the lobes of the buccal ganglia was routinely removed. Occasionally the inner transparent sheath was also removed.

Isolation of the VWC soma was performed in the desheathed buccal ganglion. A cluster of 3-10 somata including the VWC was cut away from the neuropil using fine scissors. In three of four cases, all adhering somata were gently teased off the VWC with fine tweezers; direct microscopic observation showed VWC axon stubs of less than $50 \mu \mathrm{m}$ in length. In the fourth case adhering somata were not removed; however, postexperimental cobalt injection and development showed an axon stub of less than 50 $\mu \mathrm{m}$ and no evidence of dendritic processes. Thus, isolation was virtually complete in this case. The isolated somata were stabilized against two insect pins for intracellular recording.

\section{RESULTS}

The results are presented in three sections. In the first, we report data on the morphology of the VWCs, as determined by cobalt staining. The second section shows that the command ability of the VWC derives from broadening of action potentials that occurs during endogenously sustained VWC bursts. The third section summarizes what is known about the synaptic physiology of the VWCs. 


\section{Morphology and peripheral innervation}

The bilaterally paired ventral white cells are named for their somata locations on or just beneath the ventral surface of each buccal ganglion (Fig. 1) and their whitish color. The whitish color contrasts with the orange pigmentation of surrounding somata and allows ready visual identification.

Electrophysiological mapping (intracellular soma recordings combined with extracellular recordings and orthodromic and antidromic stimulation) showed that each VWC sends an axon out on the contralateral stomatogastric nerve to ramify over the surface of the contralateral esophagus. Spontaneous VWC action potentials or those driven by intracellular stimulation are followed by longitudinal contracture of the contralateral side of the esophagus, suggesting a motoneuronal role for the VWC in addition to the interneuron function documented below.
The detailed morphology of the VWC has been examined in three ganglia by backfilling its stomatogastric axon with cobalt chloride, and in one ganglion by intracellular cobalt injection into the soma (Fig. 1). The drawing of Fig. 1 was traced from photographs of a whole mount, and fine details were added during direct microscopic observation. The single axon emerges from the large $(200 \mu \mathrm{m})$ soma and narrows to a diameter of $10-15 \mu \mathrm{m}$ in the neuropil. The majority of the fine, varicose dendritic processes branch off close to the soma; most of them ramify from two large primary dendrites near the soma. The VWC axon traverses the commissure to the contralateral side of the buccal ganglion and expands abruptly to a diameter of $25-30 \mu \mathrm{m}$. Some additional fine processes sprout from the expanded regions. The axon then contracts to about $10 \mu \mathrm{m}$ before entering the contralateral stomatogastric nerve and traveling to the periphery.

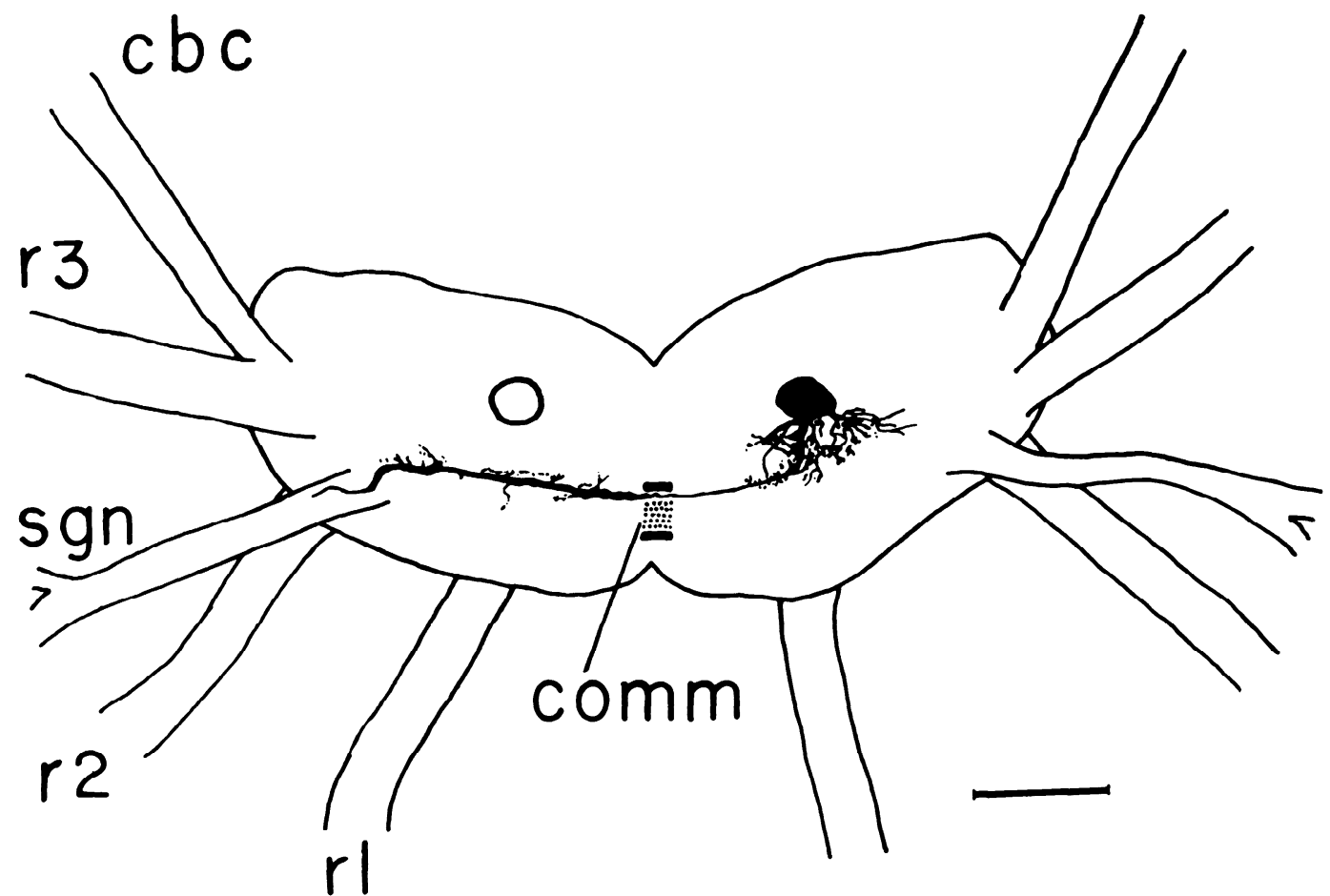

FIG. 1. Tracing of a photograph of a cobalt-stained ventral white cell in the buccal ganglion. Note dendritic fields ncar the soma, axon that extends contralaterally to exit via the stomatogastric nerve, and expanded diameter of contralateral axon segment. The position of the soma of the contralateral homologous VWC is indicated. cbc, cerebrobuccal connective; sgn, stomatogastric nerve; r1, r2, and r3, nerve roots innervating musculature of the buccal mass; comm, commissure between the paired lobes of the ganglion. Calibration bar is $500 \mu \mathrm{m}$. See text for further details. 


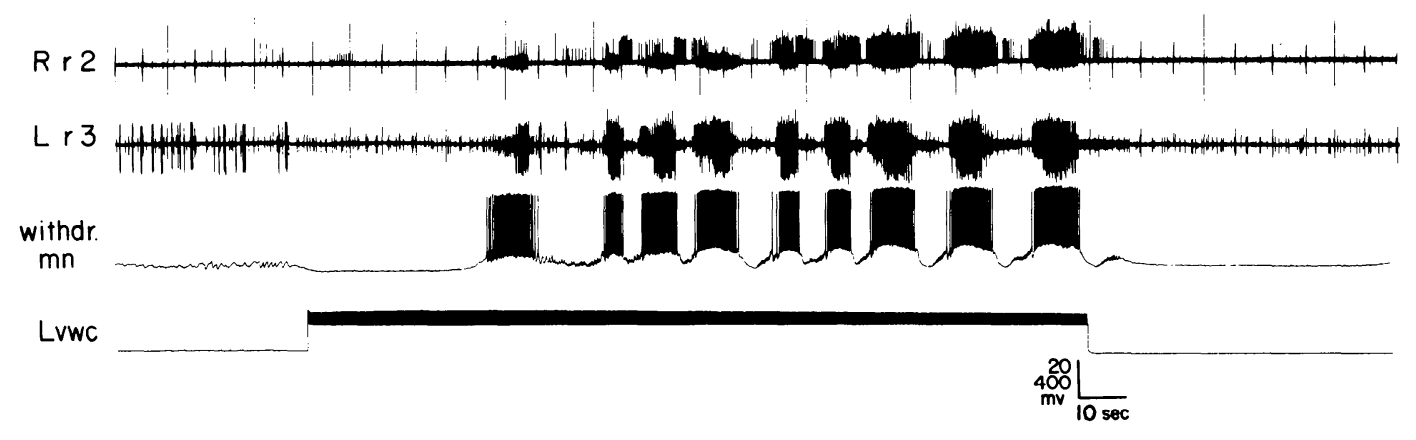

FIG. 2. Intracellular stimulation of the left ventral white cell (Lvwc) drives the feeding rhythm, recorded intracellularly from a withdrawal motoneuron (withdr $\mathrm{mn}$ ) and extracellularly from roots 2 and 3 of the buccal ganglion $(\operatorname{Rr} 2, \mathrm{Lr} 3)$.

\section{Command ability of $V W C$}

When feeding, the hungry intact Pleurobranchaea commonly shows cyclic biting and swallowing movements at rates of $0.1-$ $0.3 \mathrm{~Hz}$. Previous papers have demonstrated that the feeding rhythm of the animal can be elicited in an isolated nervous system (e.g., Refs. 5, 10). The rhythm consists of alternating bursts of activity in two antagonistic populations of feeding neurons of the brain and buccal ganglion: "eversion" neurons, which fire before and during radular protraction, and "withdrawal" neurons, which fire during the retraction phase of the cycle. In the present work we found that a similar rhythmic motor output pattern can be induced by intracellular stimulation of a single VWC at 3-5 Hz (Fig. 2). The rhythm typically commences with a latency of 10-20 s and terminates shortly after cessation of stimulation. All of the $178 \mathrm{VWCs}$ examined showed this capacity to elicit feeding output during intracellular stimulation.

In spontaneously active buccal ganglia, activity recorded intracellularly from the VWCs usually shifted between two distinct modes: brief bursts (measured in seconds) that were phase-locked to the withdrawal portion of the feeding cycle, and longer bursts (measured in minutes) that recurred at regular long intervals. Figure 3 illustrates the transition from the first to the second type of burst activity. Nearly all of the VWCs examined showed the first type of bursting (Fig. 3); $136(76 \%)$ of the VWCs examined showed the second type of bursting. In these cases large and prolonged depolarizations (15-20 mV, 1-4 min) recurred spontaneously at intervals of 3-30 min. Within individual preparations these parameters

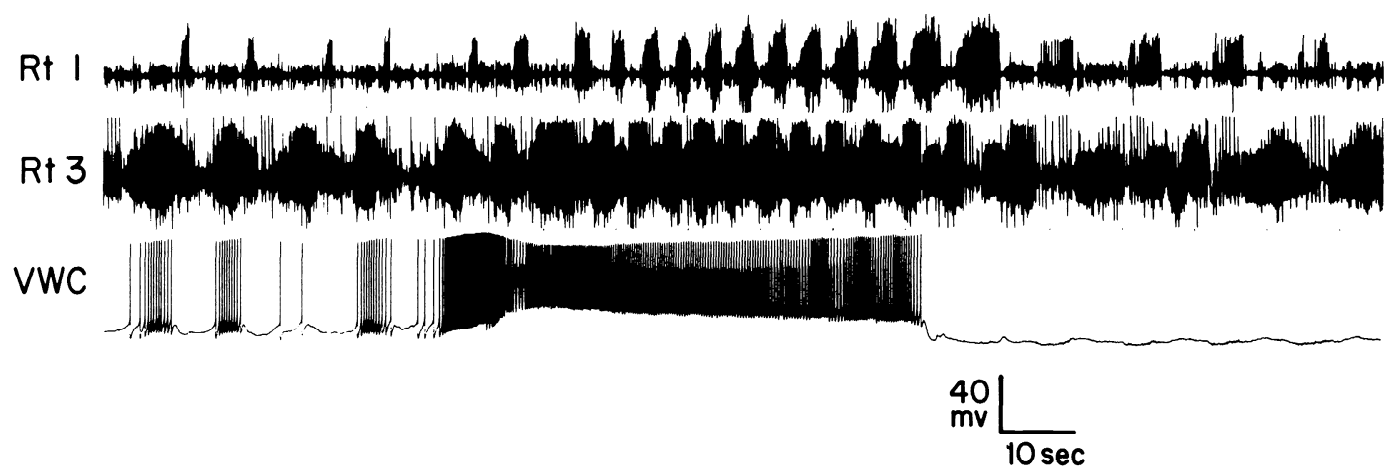

FIG. 3. Spontaneous feeding activity recorded from an isolated buccal ganglion preparation illustrating the two types of burst activity typically seen in VWC. Upper two records are extracellular recordings from roots 1 and 3 of the buccal ganglion, while lower record is an intracellular recording from the ipsilateral VWC. Typical transition from short bursts phase-locked to feeding to longer burst accompanied by intensified feeding activity is illustrated. 
were typically quite constant, though substantial variation was seen from one preparation to the next. The episodes of prolonged bursting usually appeared to be triggered off of the excitatory synaptic activity occurring during the withdrawal phase of the spontaneous feeding rhythm (Fig. 3). During these longer bursts the cyclic VWC activity described above was overshadowed while the neuron discharged a prolonged train of action potentials $(3-5 \mathrm{~Hz})$ (Fig. 3, middle of record). The VWC discharge during such episodes was invariably followed by a doubling or tripling in the frequency of the feeding rhythm, as well as an increase in the intensity of motor activity during each burst (Fig. 3, upper traces). These sustained depolarizations and bursts could also be triggered by 1 - to 10 -s depolarizing current pulses injected into the VWC late during the interburst interval (Fig. 4A) and also by spontaneous or stimulated EPSPs or by rebound from imposed hyperpolarization (Fig. $4 B)$. For the greater length of the observed
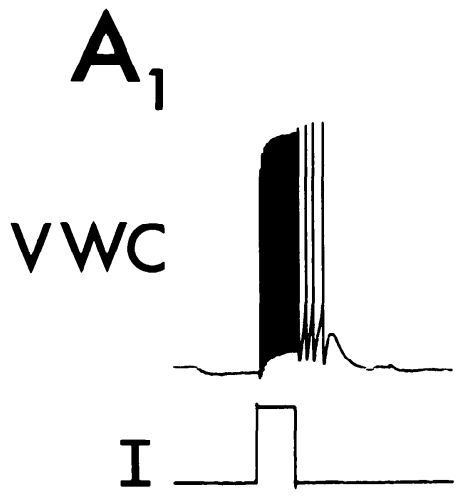

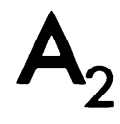

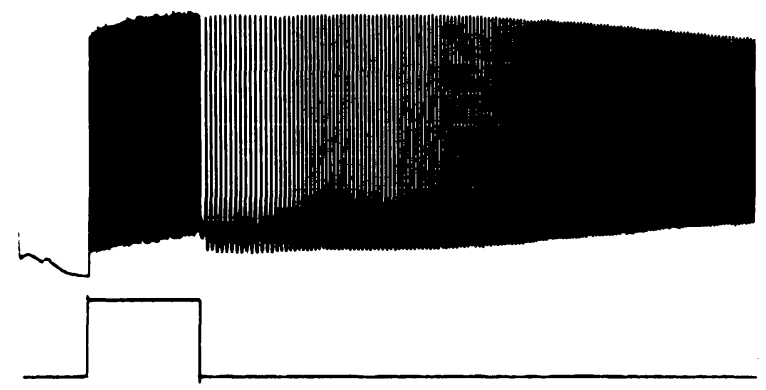
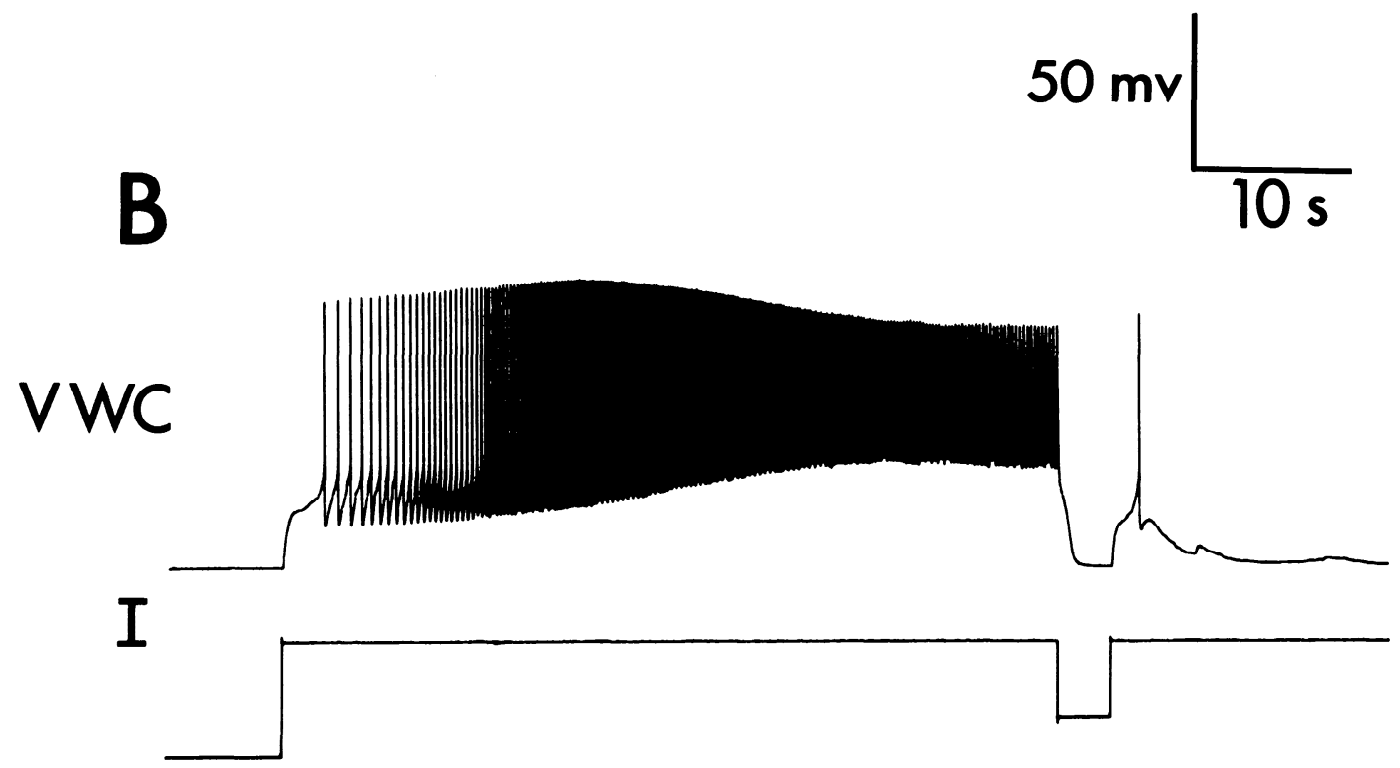

FIG. 4. Initiation and suppression of prolonged VWC bursts by current injection. $A_{1}$ : a short injection of depolarizing current initiates spikes and is followed by a depolarizing afterpotential, which supports several further spikes. $A_{2}:$ a longer current phase triggers a full prolonged burst. $B:$ a burst is initiated on rebound from imposed hyperpolarization and is terminated by a short hyperpolarizing pulse. Evidence that the current pulse terminates the burst is the lack of a characteristic slow repolarization during which spikes regain amplitude, as seen in the latter portion of the burst shown in Fig. 3. 
normal interburst interval, the triggerability showed refractoriness; that is, a pulse of depolarizing current-induced spikes and a decaying depolarizing afterpotential without triggering a full sustained burst. Bursts triggered prematurely in the cycle exhibited shorter durations. Those VWCs that did not show spontaneous episodes of prolonged bursting also did not show the triggerability, or at best fired only 1-20 spikes poststimulation.

During trains of action potentials stimulated by injected current or occurring spontaneously, the waveform of the VWC spike changed dramatically. Specifically, individual action potentials progressively broadened during repetitive discharge (Fig. 5). Spike durations typically increased from $15 \mathrm{~ms}$ (measured from takeoff) at the beginning of such trains to $75-250 \mathrm{~ms}$ during the train. Thus spike durations increased by 5to 16-fold. Associated with the increase in spike duration was a net decrease in the amplitude of the undershoot, or afterhyperpolarization, of the spike. The progressive decrement in the undershoot accompanying spike broadening contributed significantly to the average depolarization of the neuron during repetitive discharge. For instance, the undershoot decrement between the first and last spike shown in Fig. 5 is $12 \mathrm{mV}$. Broadened action potentials frequently developed a depolarizing inflection or "hump" on the repolarizing phase (Fig. 6, lower trace). The occurrence of both single and multiple humps is the reason for the irregular appearance of the spikes in the temporally compressed burst of Fig. 3 (lower trace). The hump in the somatic spikes was associated with a second spike

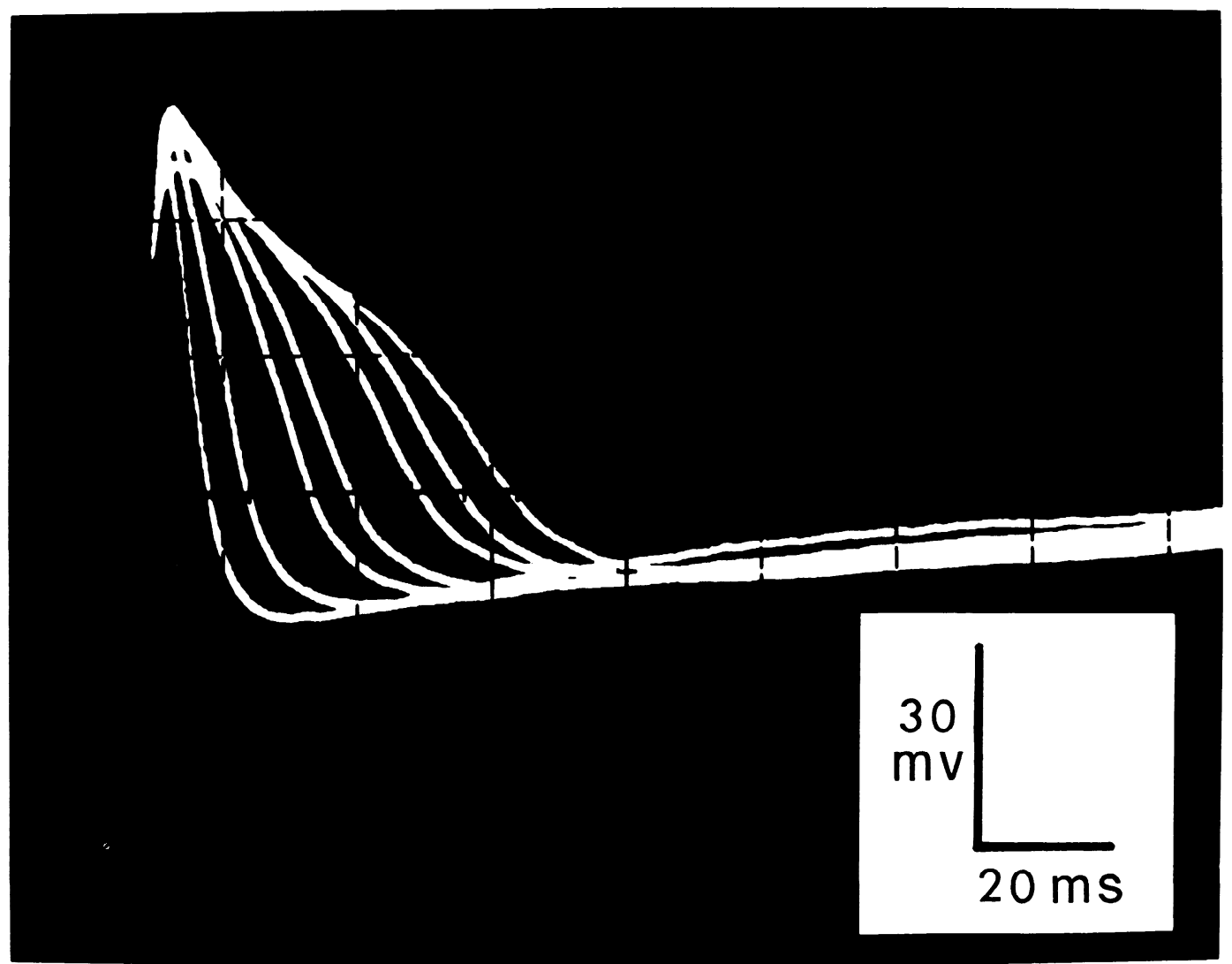

FIG. 5. Superimposed action potentials from a depolarization-induced train showing progressive spike broadening. Action potentials were driven at about $3 \mathrm{~Hz}$ by a separate current-passing electrode and samples, respectively, at $0,10,20,30,45,60$, and $90 \mathrm{~s}$. Thus, the $1 \mathrm{st}$ and approximately 30 th, 60th, 90th, 135th, 180 th, and 270th spikes of the train are depicted. 

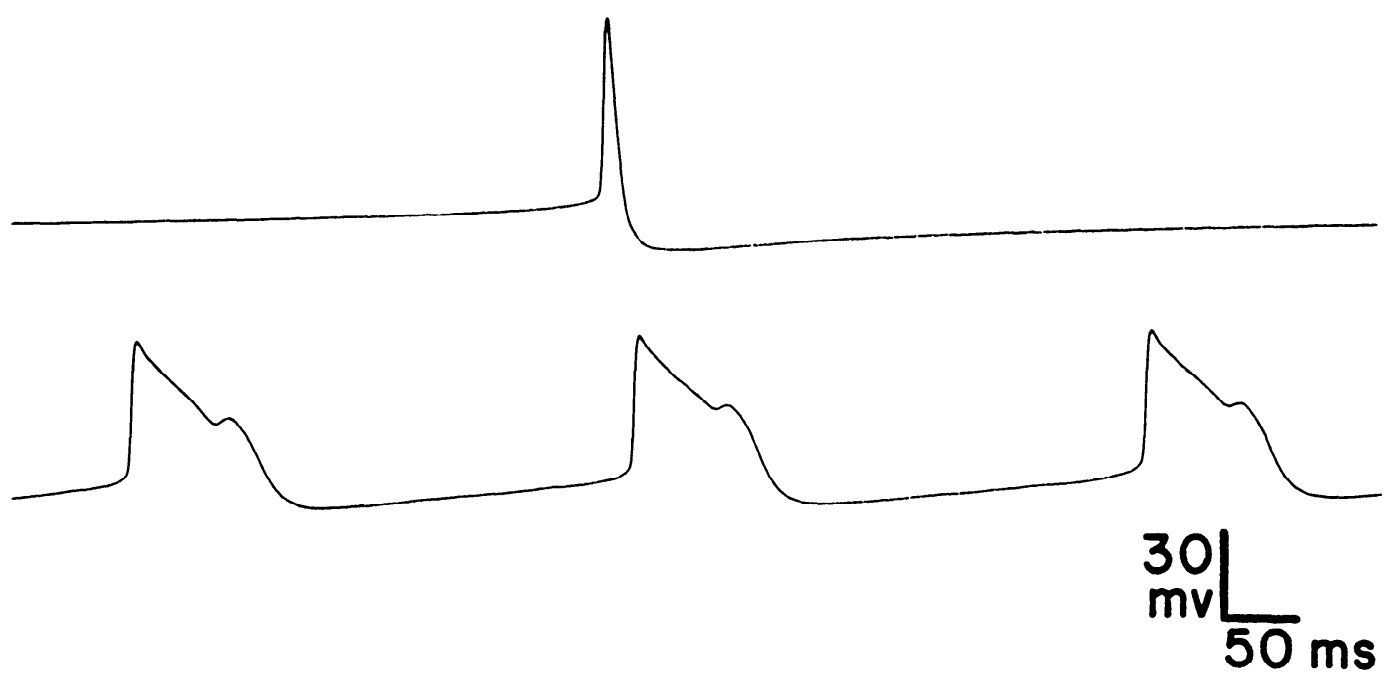

FIG. 6. Intracellular recordings of VWC action potentials before a repetitive train (upper trace) and in the latter portion of a prolonged spontaneous burst like that shown in Fig. 3. Note the distinct hump on the repolarizing phase, which occasionally develops with action-potential expansion.

recorded extracellularly in the peripheral axon (not shown). Peripheral axon spikes did not show appreciable broadening. Presumably, the hump induces the extra axon spike since broadened action potentials in the axonless isolated soma may also exhibit the hump (see further). The hump acts to prolong the somatic action potential further.

While acquiring such records, we noticed that the initiation or acceleration of the feeding rhythm coincided with the development of appreciable spike broadening, together with net membrane depolarization associated with decay of the spike undershoot. This correlation suggested that spike broadening and/or the accompanying slow depolarization increased the functional efficacy of the VWCs. We tested this hypothesis in two ways: first by assessing the effects of blockade of spike broadening and slow depolarization on feeding output, and second by ascertaining the existence of a threshold value of spike duration at which the feeding network was activated by the VWC.

In the first type of test we took advantage of the fact that spike broadening is voltage dependent. That is, it is enhanced by tonic depolarization of the soma and it is suppressed by tonically hyperpolarizing the cell. By stimulating the VWC antidromically with a suction electrode on the stomato- gastric nerve, the frequency of discharge could be held constant while the somatic spike duration was controlled by injected current. By simultaneously monitoring the feeding rhythm, the effect of spike broadening on the frequency of feeding rhythm could be directly ascertained.

Figure 7 illustrates the results of one such experiment. During a 2-min period of stimulation at $3 \mathrm{~Hz}$ in which spike broadening was permitted by imposed depolarization, 21 cycles of feeding activity occurred. When spike broadening was blocked by hyperpolarization, in an adjacent trial $5 \mathrm{~min}$ later, only 12 cycles occurred in the same time interval. For the experiment shown in Fig. 7, the average difference in the number of feeding cycles for trials of broadened spikes versus those of unbroadened spikes over 18 alternating trials was 4.22 . The difference is significant at the 0.001 level (onetailed $t$ test for matched pairs of adjacent trials). Table 1 summarizes the similar results of three such experiments on three different preparations.

Assessment of the efficacy of the VWC in this experiment. is complicated by the fact that stimulation of the stomatogastric nerve causes feeding output by itself, even when the stimulus intensity is below threshold for VWC activation. Assuming a nonlinear response curve (see Fig. 2 in Ref. 5), 


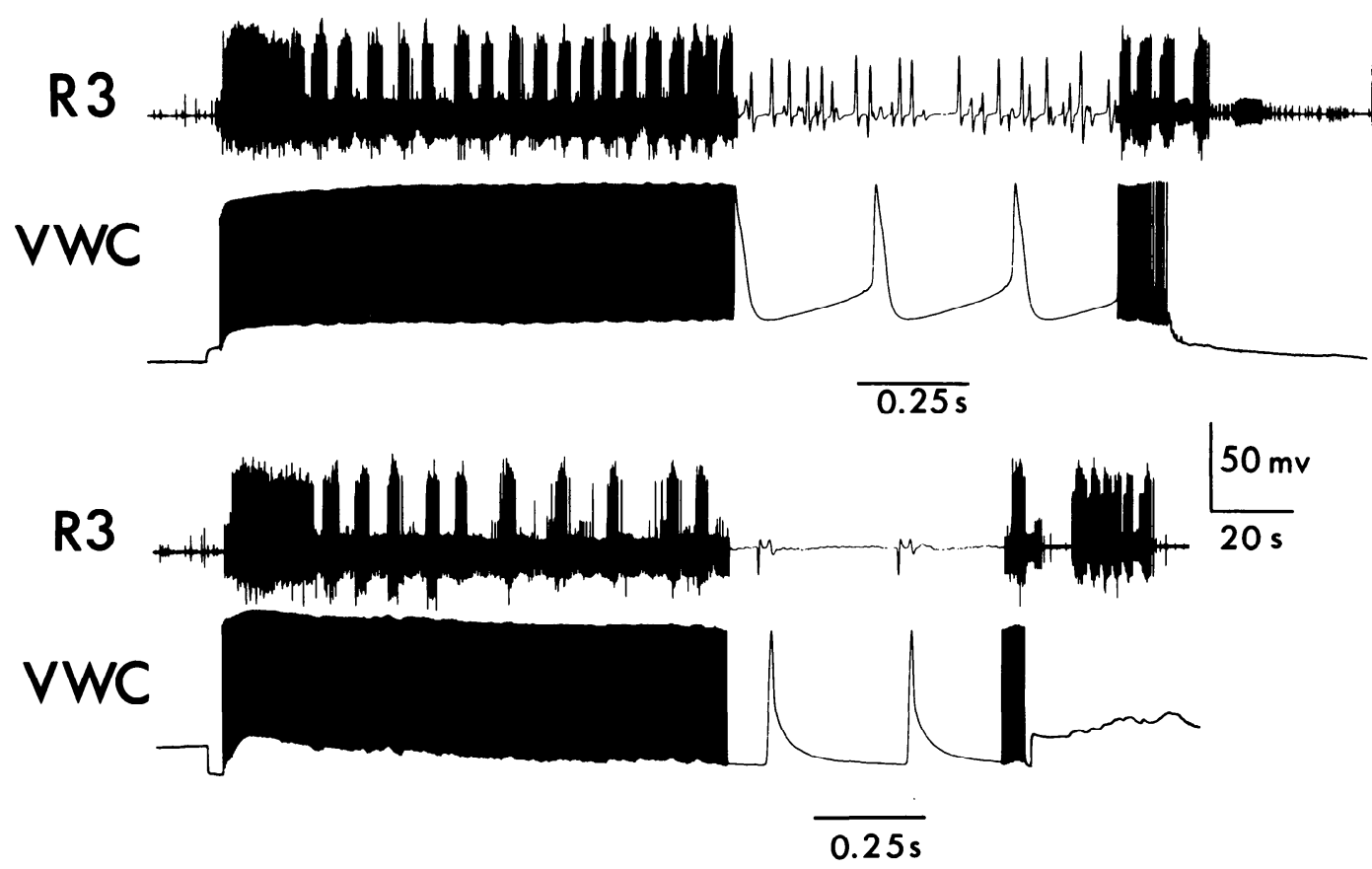

FIG. 7. Records from two adjacent trials comparing the efficacy of broadened VWC action potentials (upper records) versus unbroadened action potentials (lower records) in driving the cyclic motor output of feeding. VWC spikes were antidromically driven at $3 \mathrm{~Hz}$ for $2 \mathrm{~min}$ by stimulation of the stomatogastric nerve. Spike broadening was enhanced by depolarizing current injection in the VWC soma (upper VWC record) and suppressed by hyperpolarizing current injection (lower VWC record), while cycles of motoneuron activity were recorded extracellularly in the nerve $\mathrm{R} 3$. The chart record speed was increased transiently by $10 \times$ to show the relative waveforms of the action potentials during the latter portion of the trials. In these trials broadened spikes elicited 21 cycles of motor activity during the stimulus train, whereas unbroadened spikes were accompanied by only 12 cycles. See text.

activation of the feeding rhythm by stomatogastric nerve stimulation may partially mask the additional excitatory contribution of broadened VWC spikes.

The second type of experiment testing the efficacy of spike broadening was performed on three preparations in which spontaneous feeding activity was low enough to permit reliable measurements (about 1 cycle/3-10 min). For two of these preparations the spontaneous feeding output was reduced to an acceptable level by raising the $\mathrm{Mg}^{2+}$ concentration of the bath saline from 50 to 70 mM. A VWC was intracellularly stimulated at various levels of depolarizing current while the extent of spike broadening was recorded from a storage oscilloscope screen to the nearest millisecond. Final spike duration was measured at the half-amplitude of the initial spike of the train stimulated at the lowest current. Initial spikes were 8-10 $\mathrm{ms}$ in duration. The activity of the feeding network was recorded extracellularly from a nerve (root 3 ) of the ganglion. VWC spikes were allowed to broaden until a burst of motorneuron action potentials was initiated in the nerve. In this manner we could obtain an approximate threshold value of the spike duration necessary to initiate feeding activity that was repeatable and similar for all strengths of depolarizing currents. The results are summarized in Table 2 . In each experiment, over a wide range of depolarizing current intensities, the VWC spike duration at which motoneuron activity was initiated in the nerve invariably measured within a few milliseconds of the overall mean $(21.6 \mathrm{~ms})$. The rate of spike broadening during a train is largely dependent on the level of depolarizing stimulus current (unpublished observations). Thus, average spike frequencies measured between 2.5 and $2.9 \mathrm{~Hz}$ at the lower stimulus currents and between 4.1 and $6.0 \mathrm{~Hz}$ at the higher cur- 
TABLE 1. Efficacy of broadened vs. unbroadened spikes in driving feeding output

\begin{tabular}{|c|c|c|c|c|c|}
\hline \multirow[b]{2}{*}{ Expt } & \multicolumn{2}{|c|}{ No. of Trials } & \multicolumn{2}{|c|}{$\begin{array}{l}\text { Avg No. of Feeding } \\
\text { Cycles Stimulated }\end{array}$} & \multirow[b]{2}{*}{ Significance Level, $P$} \\
\hline & $\begin{array}{l}\text { Broadened } \\
\text { spikes }\end{array}$ & $\begin{array}{l}\text { Unbroadened } \\
\text { spikes }\end{array}$ & $\begin{array}{l}\text { Broadened } \\
\text { spikes }\end{array}$ & $\begin{array}{l}\text { Unbroadened } \\
\text { spikes }\end{array}$ & \\
\hline 1 & 8 & 8 & 19.13 & 14.63 & $<0.005$ \\
\hline 2 & 6 & 6 & 21.33 & 16.83 & $<0.025$ \\
\hline 3 & 9 & 9 & 19.75 & 15.38 & $<0.001$ \\
\hline
\end{tabular}

Results of experiments on three different preparations comparing the efficacy of broadened vs. unbroadened VWC action potentials in driving the output of the feeding motor network. VWC spikes were stimulated antidromically at $3 \mathrm{~Hz}$, while spike broadening was either enhanced or suppressed by intrasomatic current injection in alternating trials separated by 5 -min intervals. The activity of the contralateral (unrecorded) VWC was controlled for in experiment $l$ by surgically destroying its soma; after an initial injury burst of axon spikes recorded extracellularly in its nerve, no further activity was recorded. In experiments 2 and 3 , both VWCs of the ganglion were antidromically stimulated, while spike broadening of only one was controlled by injected current. In the data analysis trials of broadened spikes were paired with adjacent trials of unbroadened spikes. The results indicate that trains of broadened spikes are significantly more effective in driving motor output than are trains of unbroadened spikes. $P$ values obtained by one-tailed $t$ test.

rents, while the spike train durations ranged between 30 and $150 \mathrm{~s}$ and 9 and $22 \mathrm{~s}$ for lowest and highest currents, respectively. Figure 8 shows records from these experiments. The records of Fig. $8 A$ and $B$ compare feeding burst initiation at stimuluscurrent intensities of 2.4 and $5.3 \mathrm{nA}$, respectively. In the first test shown in Fig. 8A, the stimulus current was stopped when spike duration reached $19 \mathrm{~ms}$, a duration that did not elicit activity in the feeding nerve (lower trace). However, when the spike duration was allowed to expand to $20 \mathrm{~ms}$ (second spike train, middle of the record) motoneuron activity was initiated. Similarly in Fig. $8 B$, feeding activity occurred when spike duration was recorded at $23 \mathrm{~ms}$ in the three trials shown. These latter values are likely to be high due to the high spike frequency and rapid broadening.

The experiments described above show both that broadened spikes are more effective than unbroadened spikes in driving the feeding network and that in a given preparation, a certain minimum VWC spike duration may be necessary for its effectiveness in driving the network to be attained, regardless of the depolarizing stimulus. While these experiments do not directly assess the possible contribution of the depolarizations from which spikes are generated due to the uncontrolled spike frequencies, in view of the rather exact thresholds of effective spike duration at all current strengths, this contribution is likely to be small compared to that of the broadened spike itself.

In order to separate endogenous expression from presynaptic influences, 19 attempts were made to dissect the VWC soma from the ganglion and study it in isolation. Of these, only four cell bodies retained high resting potentials $(60-65 \mathrm{mV})$ and the capacity to generate action potentials. Overshooting action potentials occurred in the isolated somata only when the membrane was depolarized by $25-30 \mathrm{mV}$. As in the intact neuron, action potentials recorded from the isolated VWC somata showed marked broadening on repetition and also developed a hump on the repolarizing phase (Fig. 9). Unlike the intact VWC, action potentials stimulated in the isolated somata emerged gradually out of regular oscillations of the membrane potential (Fig. 9, record 1), a result that possibly reflects differences in the somatic and axonal membrane.

All four isolated somata demonstrated sustained depolarization and repetitive spiking that could be triggered by a depolarizing stimulus. Figure 10 shows a typical prolonged burst in an isolated soma triggered by a 40 -s depolarizing stimulus. The burst outlasted the triggering stimulus by $2 \mathrm{~min}$ and $32 \mathrm{~s}$. The record of Fig. 10 may be compared with that from an intact VWC in Fig. $4 A_{2}$, which is similar except for the gradual emergence of overshooting spikes in 
TABLE 2. VWC broadened spike duration initiating feeding burst

\begin{tabular}{ccc}
\hline \hline Expt & $\begin{array}{c}\text { Stimulation } \\
\text { Current, nA }\end{array}$ & $\begin{array}{c}\text { VWC Spike Duration at } \\
\text { Feeding Burst, ms }\end{array}$ \\
\hline$l$ & 2.4 & $22.29 \pm 1.35(7)$ \\
& 2.7 & $19.50 \pm 0.71(2)$ \\
& 3.0 & $22.00 \pm 1.73(3)$ \\
& 3.4 & $24.10 \pm 1.95(5)$ \\
& 3.6 & $24.50 \pm 2.12(2)$ \\
2 & 5.3 & $23.20 \pm 1.79(5)$ \\
& 4.0 & $21.16 \pm 1.47(6)$ \\
& 4.75 & $20.67 \pm 0.82(6)$ \\
3 & 5.25 & $20.00 \pm 0.63(6)$ \\
& 6.0 & $20.00 \pm 0.63(6)$ \\
& 2.0 & $22.33 \pm 0.82(6)$ \\
& 2.5 & $21.50 \pm 1.22(6)$ \\
& 3.0 & $20.83 \pm 0.75(6)$ \\
& 3.5 & $20.67 \pm 0.52(6)$ \\
& 4.0 & $21.33 \pm 0.52(6)$ \\
\hline
\end{tabular}

Values are means \pm SD. Figures in parentheses are $n$. Results of three experiments on different preparations ascertaining the VWC spike duration at which feeding network activity was initiated. Trains of progressively broadening VWC spikes were driven at different strengths of depolarizing current while motoneuron activity was recorded in a feeding nerve. The first experiment was performed with a ganglion that showed a relatively low frequency of spontaneous motoneuron bursts (about $0.3 / \mathrm{min}$ ). In the second and third experiments, spontaneous activity was reduced by raising bath $\mathrm{Mg}^{2+}$ by $20 \mathrm{mM}$. This procedure also seemed to reduce the variance in the VWC spike duration necessary to induce motoneuron activity. See text for additional detail. The repeatability of the value of the effective VWC spike duration at all strengths of depolarizing current stimuli confirms the effectiveness of broadened action potentials and suggests that the contributory effects of the depolarizations from which the action potentials arise are relatively small.

the isolated soma and the duration of the triggering stimulus. One of the isolated somata showed the capacity for sustaining poststimulus activity only weakly, at best firing spikes of decreasing amplitude at 0.75 $\mathrm{Hz}$ for $20 \mathrm{~s}$ poststimulation. As in intact VWCs, the triggerability of prolonged bursts in the isolated somata showed refractoriness for a period (20-30 min) following a burst.

\section{Synaptic physiology of $V W C$}

Despite an intensive exploration of the identified classes of neurons within the feeding network, we have been unable to find definite postsynaptic targets of the VWC. Classes of neurons sampled in this unsuccessful search include the powerful interneurons, the anterior-ventral (AV) cells (10) (30 penetrations in 12 preparations); another identified bilateral pair of commandlike neurons in the buccal ganglion termed the B3 interneurons (1 penetration in 1 preparation); withdrawal motoneurons (10 penetrations in 5 preparations); eversion motoneurons ( 6 penetrations in 5 preparations); putative sensory neurons (17) (10 penetrations in 3 preparations); corollary discharge neurons ( 2 penetrations in 2 preparations); and the efference copy neurons (5) (2 penetrations in 2 preparations). It would appear that either a major class of feeding neurons remains undiscovered or the VWC exerts its effect in some way other than conventional postsynaptic potentials.

In contrast to the elusive postsynaptic effects of the VWC, one electrically coupled and various chemically presynaptic neurons were readily found. Each VWC is strongly coupled to its contralateral homolog by presumed electrical synapses (Fig. 11). Oscillatory potentials imposed on the soma of one member of the pair by current injection are recorded in the contralateral partner as attenuated oscillations; action potentials stimulated in one caused biphasic inflections of 4- to 5-mV amplitude in the partner with negligible latency (Fig. 11). As would be expected from such coupling, the paired VWCs show a high degree of synchrony in spontaneous fluctuations of their membrane potentials (Fig. 12A), in the times of onset and termination of spontaneous prolonged bursting episodes (Fig. 12B), and in the occurrence of spikes during the burst (Fig. 12C).

Numerous synaptic inputs to the VWC have been found from other identified members of the feeding network. In fact, practically every known feeding interneuron tested exhibited a synaptic effect on the VWC. Of 22 anterior-ventral neurons tested in 12 preparations, 16 caused EPSPs $(0.5-$ $10 \mathrm{mV})$ and 6 caused IPSPs $(0.5-5 \mathrm{mV})$ in the VWC. The paracerebral neurons provide an example whose role in behavior has been characterized. These cells, whose somata are located in the brain and send descending axons to the buccal ganglion, were previously shown to serve as command neurons for the feeding behavior (10). The 

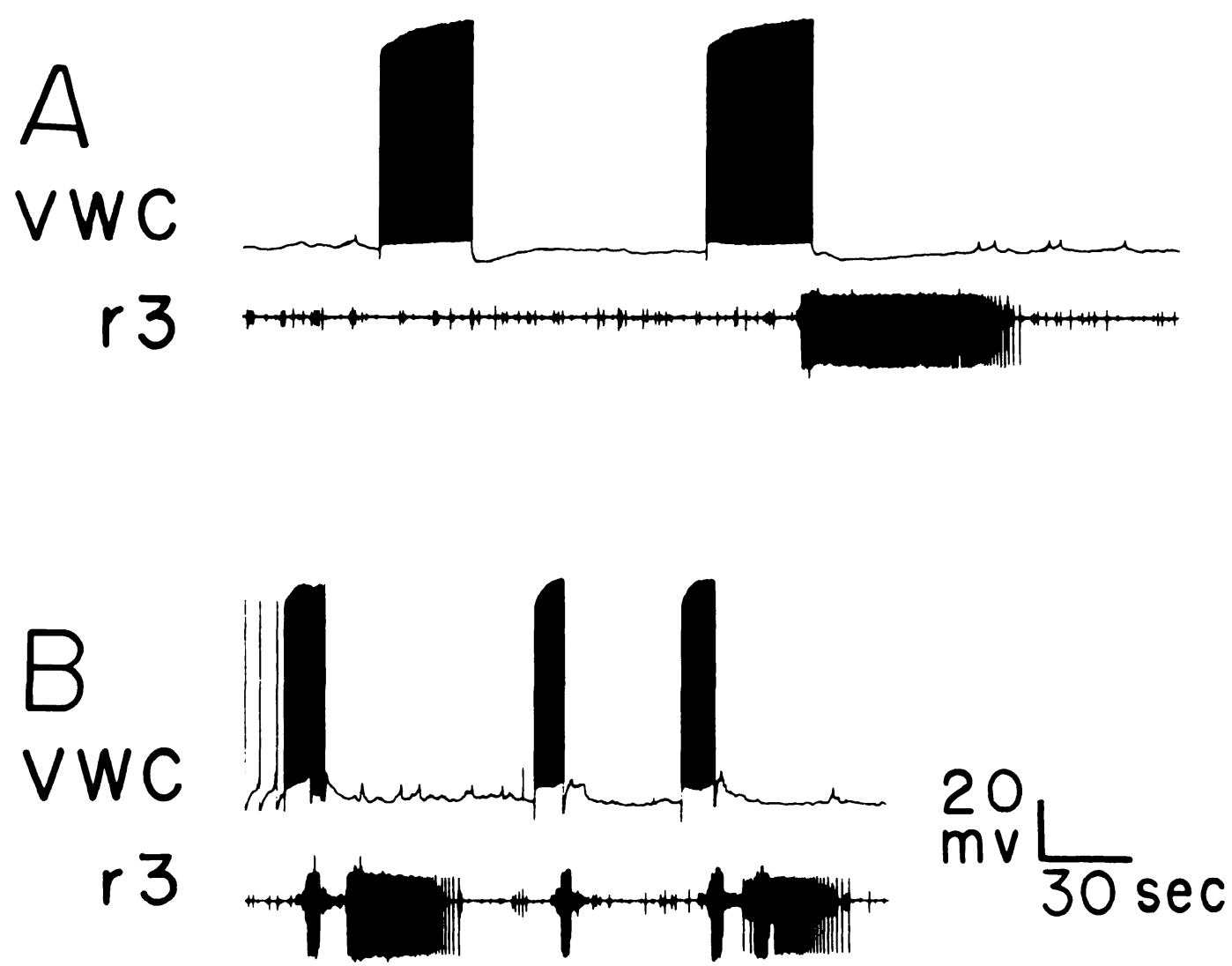

FIG. 8. Current-stimulated trains of progressively broadening action potentials in a VWC initiate motoneuron bursts in a nerve ( $\mathrm{r} 3$ ) only on reaching action-potential durations of about $20 \mathrm{~ms}$. $A$ : depolarizing stimulus currents $2.4 \mathrm{nA}$. The first stimulus train is terminated when spike duration attains $19 \mathrm{~ms}$. Motoneuron activity is only initiated when, in the second train, spike durations attain $20 \mathrm{~ms} . B$ : depolarizing stimulus currents $5.3 \mathrm{nA} ; 1-2$ cycles of motoneuron bursts are initiated at spike durations of $23 \mathrm{~ms}$ in the three trials shown. While the appearance of the motoneuron bursts changed somewhat during this prolonged experiment, the VWC spike-duration threshold did not (see Table 2, expt 1 ).

paracerebral neurons normally fire bursts of action potentials during radular eversion, and they supply long-latency $(400 \mathrm{~ms})$, relatively slow (Fig. 13A) and summating (Fig. 13B ) IPSPs to both VWCs. Since the VWCs fire during the withdrawal phase of feeding, the phasic inhibition from the paracerebrals during eversion is in accord with the typical cyclic discharge pattern of the VWCs.

An example of excitatory inputs to the VWCs is from another pair of neurons of the buccal ganglion with the ability to drive feeding (unpublished observations), the B3 interneurons (so named for their ventral position in rectangular coordinates (17)). These inputs consist of discrete and facilitating, short-latency EPSPs (Fig. 13C). The
B3 interneurons fire normally during the withdrawal phase of feeding; therefore, the excitation they provide the VWCs may also contribute to the cyclic discharge pattern of the VWCs during feeding output.

\section{DISCUSSION}

\section{Morphology of ventral white cell}

The geometry of the VWC, as visualized by intracellular cobalt staining, exhibits two structural features that are potentially relevant to its function. First, the threefold expansion of axon diameter as it courses through the contralateral buccal ganglion in the region of the major dendritic field of its bilateral homolog (Fig. 1) furnishes a possible anatomical site for the presumed 

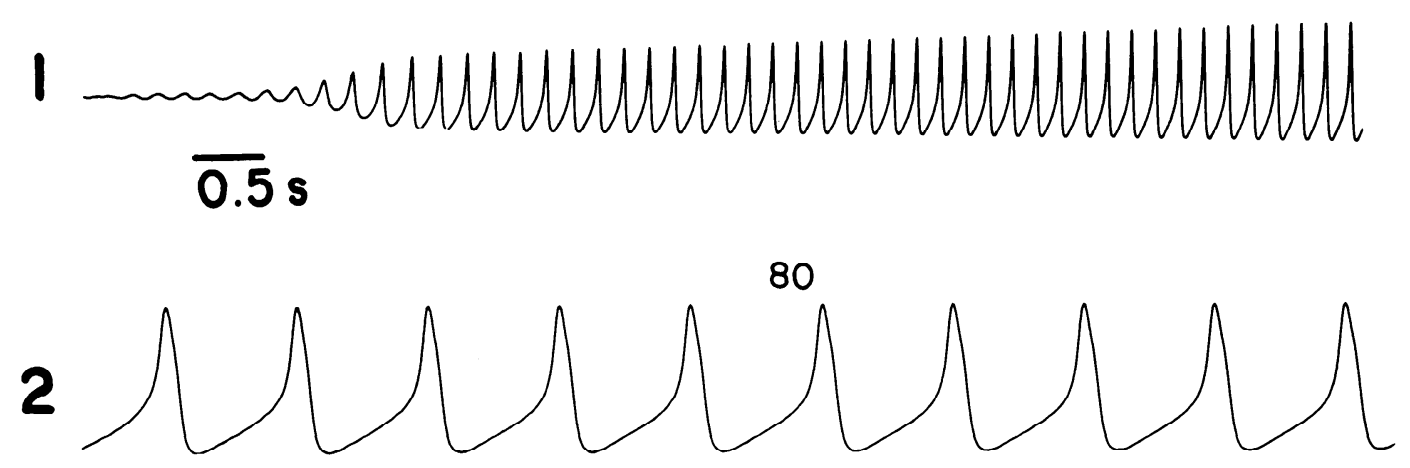

\section{1 mv $100 \mathrm{~ms}$}
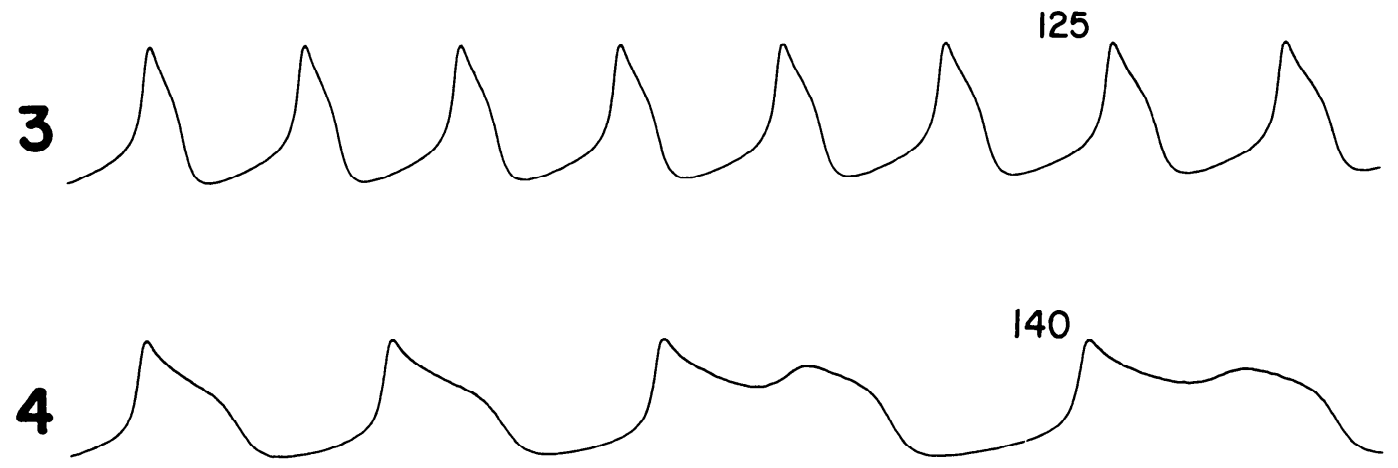

FIG. 9. Broadening of action potentials in a train stimulated by intracellular injection of depolarizing current in the surgically isolated VWC soma. Record 1 shows the gradual development of full-sized action potentials from regular oscillations in the potential of the depolarized membrane. Records 2-4 illustrate the progressive broadening of the action-potential duration during repetitive activity. Record 4 shows the development of a depolarizing hump on the repolarizing phase. Small numbers refer to the serial-order number of the action potentials in the train.

electrical coupling between the two cells. Gap junctions, the morphological substrates of electrical coupling, have been localized at axodendritic sites in other gastropod central neurons $(7,11)$ and may form the basis of the functional coupling between the VWCs demonstrated here (Fig. 11). In this case the expanded axonal segment may act as a significant current source to the contralateral homolog, contributing to the observed functional synchrony. Synchronous discharge of the two ventral white cells may in turn contribute to bilateral coordination of the esophagus during feeding behavior.

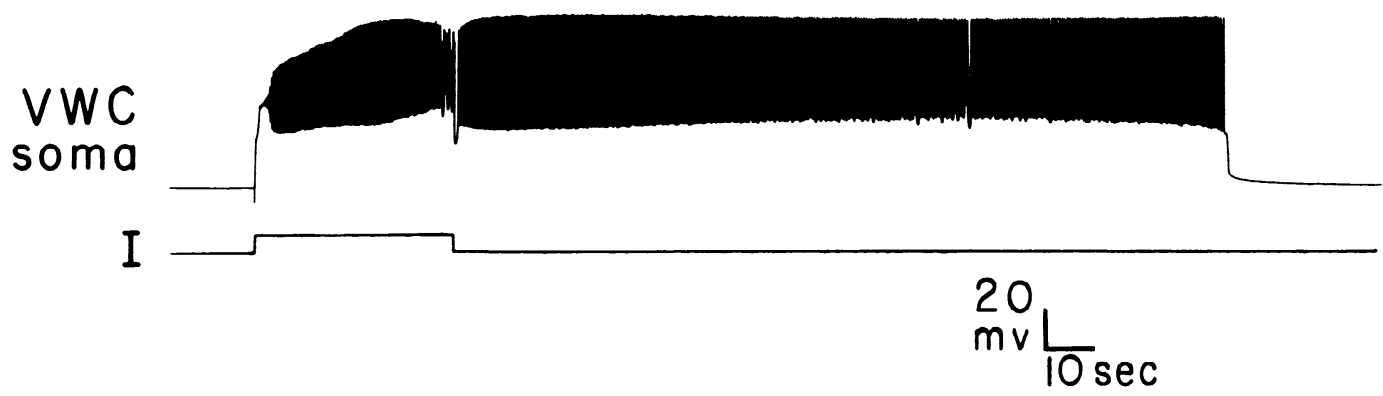

FIG. 10. A sustained-burst episode triggered in the isolated VWC cell body by depolarizing current. 

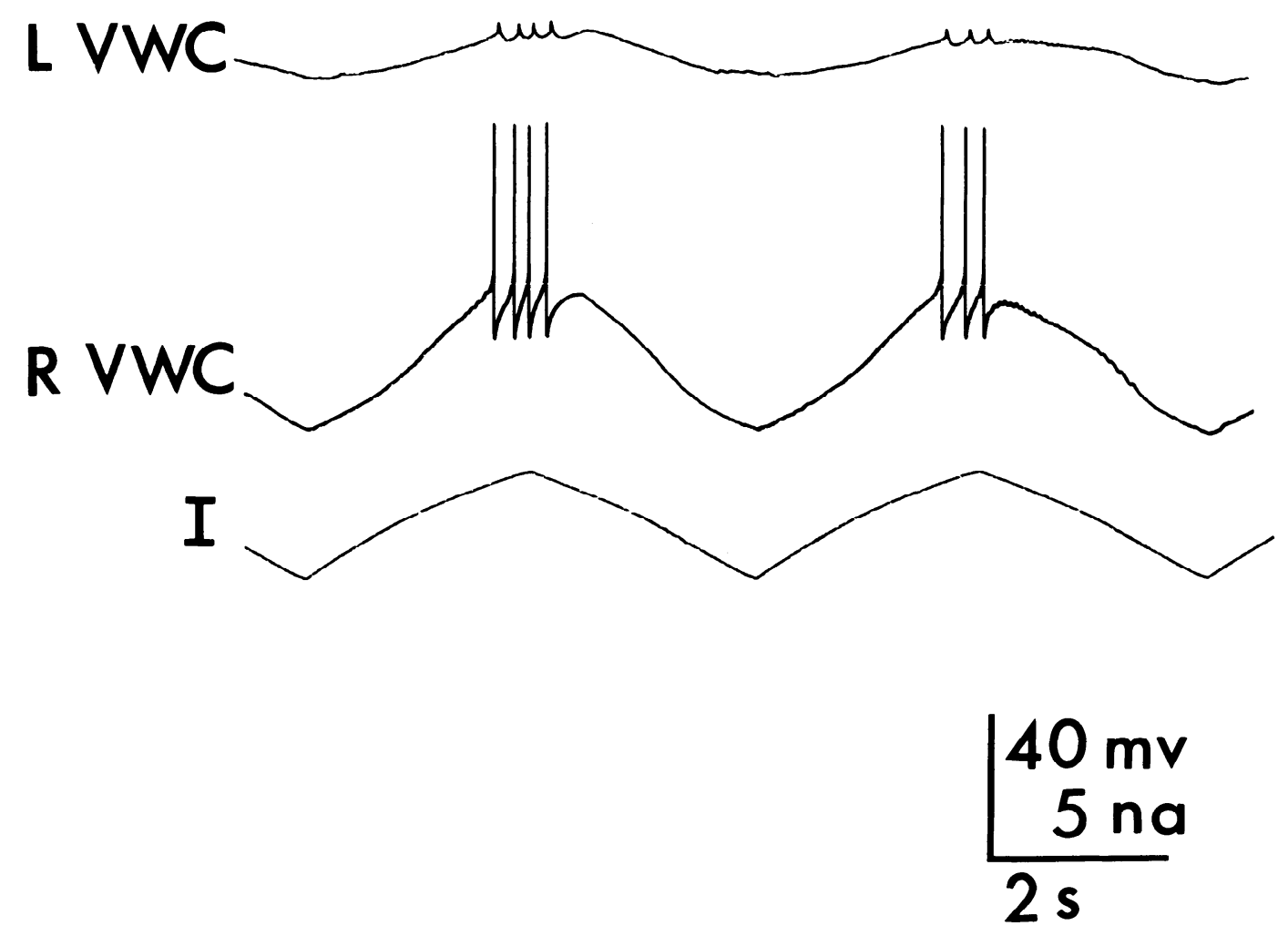

FIG. 11. Electrical coupling of the VWCs. Regular oscillations of the soma potential of the right VWC induced by injected current (I) cause attenuated oscillations in the partner in the left ganglion lobe.

A second morphological feature with possible functional implications is the close physical proximity to the VWC soma to its dendritic field. Cobalt injection has shown that the major dendritic fields of the VWC branch off near the soma and extend to a few hundred micrometers of the soma. Combined physiological and morphological studies on other gastropod neurons suggest space constants of several millimeters (12). Therefore, the dendrites of the VWC may be located within a space constant of the soma. By analogy with earlier studies on molluscan neurons, the dendrites in turn presumably represent the major synaptic input-output sites of the $\operatorname{VWC}(7,11,12,19)$. Therefore, the broadened action potentials occurring in the VWC soma would be expected to exert a controlling influence on the synaptic output of the VWCs.

\section{Functional role of broadened action potentials}

Progressive spike broadening with repetition has been demonstrated previously in molluscan neurons $(6,20)$, but a functional role has not been previously demonstrated. In the present work we have shown that spike broadening in the VWC is invariably correlated with acceleration of the feeding rhythm (Fig. 3). Direct manipulation of the degree of spike broadening by controlling the membrane voltage (Fig. 7) suggests further that spike broadening is directly causal to augmented feeding output. The finding that the initiation of activity in the feeding network is associated with a rather precise threshold duration of the VWC spike regardless of the level of the depolarizing stimulus current (Table 2, Fig. 8), both confirms the effectiveness of the broadened spike and provides evidence that the contribution of the tonic membrane depolarization driving repetitive spiking is itself negligible.

\section{Mechanism of effects of broadened action potentials}

Despite an intensive search, we have not been able to identify sites of direct post- 


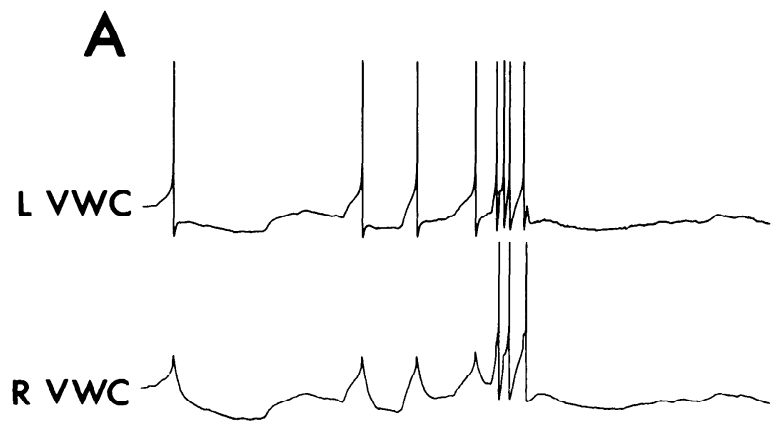

B
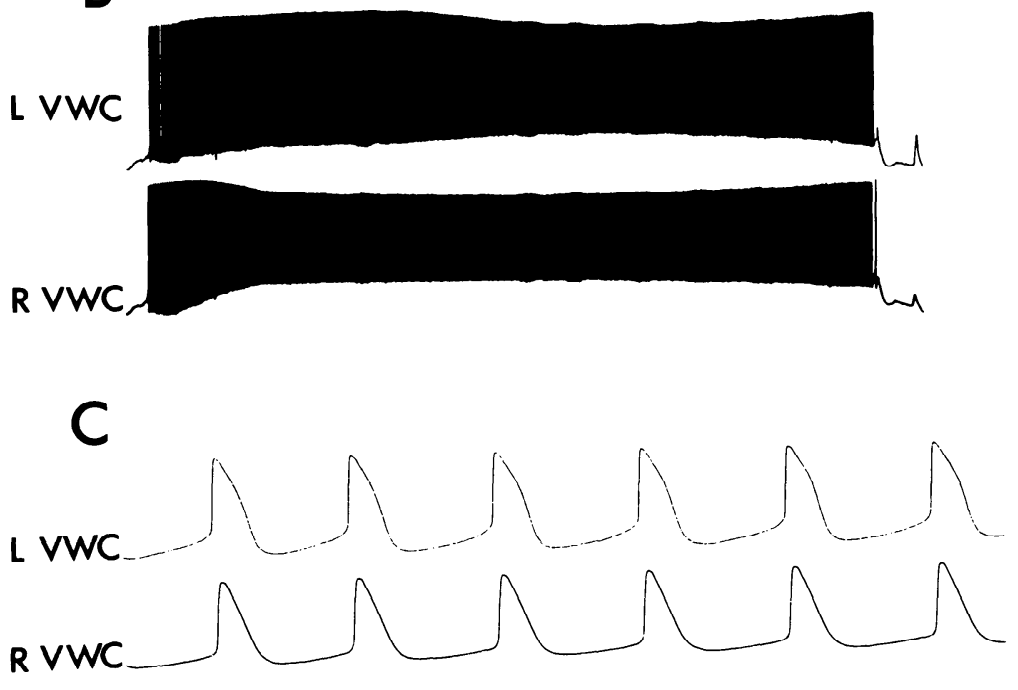

$$
\begin{array}{ll}
25 \mathrm{mv} & (A) \\
50 \mathrm{mv} & (B+C) \\
5 \mathrm{~s} & 25 \mathrm{~s} \\
250 \mathrm{~ms} &
\end{array}
$$

FIG. 12. Electrical coupling and synchrony of activity in the two VWCs. $A$ : synchrony of spontaneous fluctuations of membrane potentials. $B$ : synchrony of onset and termination of spontaneous burst episodes in the neurons. $C$ : synchrony of action potentials occurring during spontaneous bursts in neurons.

synaptic action of the VWCs. Such negative results suggest that the VWCs exert their effects by indirect or unconventional synaptic mechanisms, such as heterosynaptic facilitation (13) or the induction of special properties of excitability in other network members $(2,15)$. In this regard it is notable that white neurons in the related mollusk Aplysia have been implicated in the release of neuromodulatory peptides (2). Another possibility, as yet untested, is that the VWCs excite the feeding network via potassium depolarization; that is, the broadened action potentials may cause significant local release of potassium within the neuropil, resulting in depolarization and consequent activation of many members of the feeding network.

\section{Recurrent prolonged bursting in VWC}

The broadened action potentials that impart the ability of the VWC to drive the feeding network normally occur spon- 
A

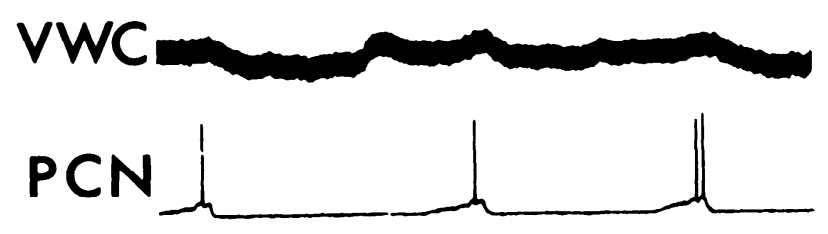

B

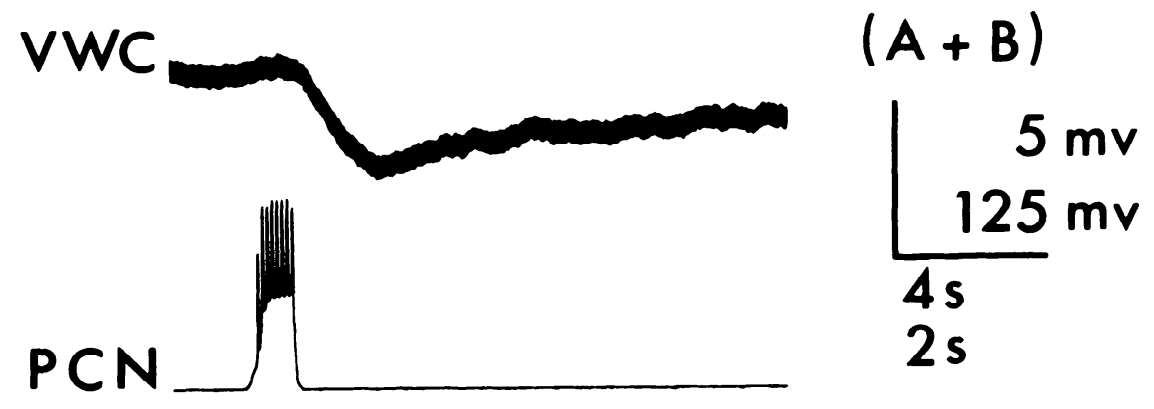

C
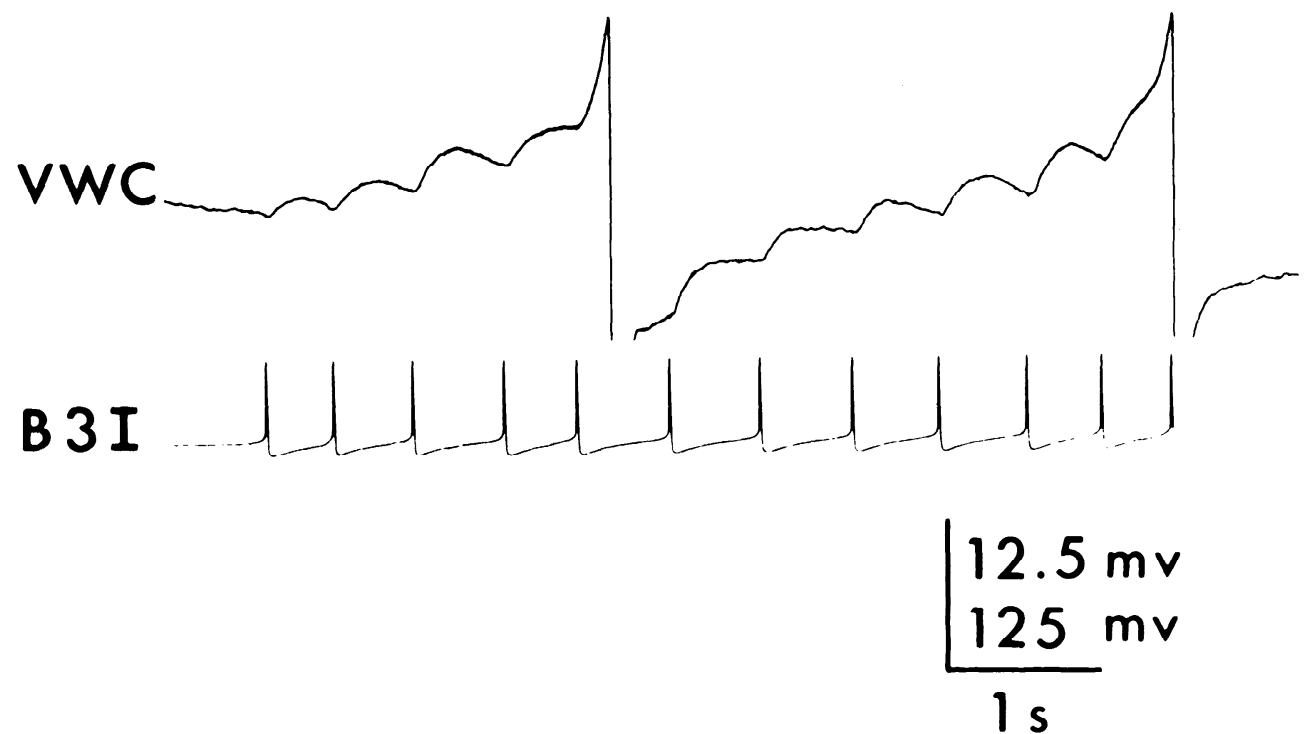

FIG. 13. Synaptic inputs to the VWC from major feeding interneurons of the network. $A$ : single currentdriven action potentials in a paracerebral cell (PCN), which has its cell body in the brain, cause slow IPSPs in the VWC. $B$ : summation of IPSPs in the VWC driven by the PCN. $C$ : one of the bilaterally paired B3 interneurons in the buccal ganglion mediates facilitating EPSPs to the VWC. Signs of the synaptic potentials are consistent with the phases of spike activity of neurons during the feeding cycle (see text).

taneously during characteristic prolonged and recurrent membrane depolarizations. The prolonged depolarizations and repeti- tive spiking can be triggered by injected current in both the intact and the isolated VWC cell body, suggesting that they are 
endogenous to the neuron. These potentials are superficially similar to those that underlie endogenous bursting in other molluscan and arthropod neurons $(1,15,16)$, with the distinction that the burst durations and interburst intervals are exceptionally prolonged. In the intact cell spontaneous synaptic inputs are apparently adequate stimuli for triggering a burst episode in the nonrefractory VWC. Whether the prolonged depolarizations may actually occur as spontaneous "pacemaker" potentials (1) or if they always require an external triggering stimulus is not yet certain due to the limited number of observations on the isolated cell body.

\section{Role of ventral white cell in feeding}

Episodes of spontaneous feeding behavior in intact animals in the absence of appropriate stimuli have been observed but rarely. The recurrent prolonged burst episodes observed in the majority of the isolated nervous systems may reflect some facet of arousal or disinhibition of the ventral white cell resulting from dissection and/or isolation of the CNS. Our results suggest that during feeding in the intact animal, burst episodes triggered in the VWCs by synaptic inputs from the motor network

\section{REFERENCES}

1. Alving, B. Difference between pacemaker and nonpacemaker neurons of neurons of Aplysia on voltage-clamping. J. Gen. Physiol. 54: 512-531, 1969.

2. Branton, W. D., Mayeri, E., Brownell, P., AND SIMON, S. B. Evidence for local hormonal communication between neurones in Aplysia. Nature London 274: 70-72, 1978.

3. Davis, W. J. and Gillette, R. Neural correlate of behavioral plasticity in command neurons of Pleurobranchaea. Science 199: 801-804, 1978.

4. Davis, W. J. AND Mpitsos, G. J. Behavioral choice and habituation in the marine mollusc Pleurobranchaea californica. Z. Vergl. Physiol. 75: 207-232, 1971.

5. Davis, W. J., Mpitsos, G. J., Siegler, M. V. S., Pinneo, J. M., AND Davis, K. B. Neuronal substrates of behavioral hierarchies and associative learning in Pleurobranchaea. Am. Zool. 14: $1037-1050,1974$.

6. ECKert, R. AND LuX, H. D. Calcium-dependent depression of a late outward current in snail neurons. Science 197: 472-475, 1977.

7. Gillette, R. Microstructural and Ultrastructural Studies on Identified Neurons of the Abdominal Ganglion of Aplysia californica (Thesis). Toronto: University of Toronto, 1974.

8. Gillette, R. and Davis, W. J. The role of the would act to initiate feeding or to intensify ongoing feeding activity. Evidence that the VWCs normally do exert a command function in behavior has so far proved difficult to obtain as the VWCs are located on the ventral side of the buccal ganglion, closely attached to the buccal mass that undergoes large movements during feeding.

In substance, the available data show that the VWC is capable of commanding the motor output underlying feeding. The evidence reported here shows that this command ability arises from progressive broadening of spikes during repetitive activity, that in turn results from an endogenous capacity for producing prolonged bursts of spikes.

\section{ACKNOWLEDGMENTS}

This study was supported by a National Institutes of Health postdoctoral fellowship and a research grant from the Graduate Research Board of the University of Illinois to R. Gillette, a research grant from the American Philosophical Society to M. U. Gillette, and National Institutes of Health Research Grants NS 09050 and MH 23254 to W. J. Davis.

Present address of R. Gillette and M. U. Gillette: Dept. of Physiology and Biophysics, 524 Burrill Hall, University of Illinois, Urbana IL 61801.

Received 6 November 1978; accepted in final form 12 September 1979.

metacerebral giant neuron in the feeding behavior of Pleurobranchaea. J. Comp. Physiol. 116: 129159, 1977.

9. Gillette, R., Gillette, M. U., and Davis, W. J. Prolonged endogenous bursting and spike broadening are substrates of command function in the feeding network of Pleurobranchaea californica. Soc. Neurosci. Abstr. 4: 1210, 1978.

10. Gillette, R., Kovac, M. P., ANd Davis, W. J. Command neurons in Pleurobranchaea receive synaptic feedback from the motor network they excite. Science 199: 798-801, 1978.

11. Gillette, R. and Pomeranz, B. Ultrastructural correlates of interneuronal function in the abdominal ganglion of Aplysia californica. J. Neurobiol. 6: 463-474, 1975.

12. Graubard, K. Voltage attenuation with Aplysia neurons: the effect of branching pattern. Brain Res. 88: 325-332, 1975.

13. Kandel, E. AND TAUC, L. Mechanism of heterosynaptic facilitation in the giant cell of the abdominal ganglion of Aplysia depilans. J. Physiol. London 181: 28-47, 1965.

14. Pitman, R. N., Tweedle, C. W., and Cohen, M. J. Branching of central neurons: intracellular cobalt injection for light and electron microscopy. Science 176: 384-385, 1972.

15. Russel, D. F. And Hartline, D. K. Bursting 
neural networks: a reexamination. Science 200: 453-456, 1978.

16. Selverston, A. I. Structural and functional basis of motor pattern generation in the stomatogastric ganglion of the lobster. Am. Zool. 14: 957-972, 1974.

17. Siegler, M. V. S. Motor neurone coordination and sensory modulation in the feeding system of the mollusc Pleurobranchaea californica. J. Exp. Biol. 71: 27-48, 1977.

18. Siegler, M. V. S., Mpitsos, G. J., and Davis, W. J. Motor organization and generation of rhythmic feeding output in the buccal ganglion of Pleurobranchaea. J. Neurophysiol. 37: 1173$1196,1974$.

19. Thompson, E. B., Schwartz, J. H., AND KANDEL, E. R. A radioautographic analysis in the light and electron microscope of identified Aplysia neurons and their processes after intrasomatic injection of $\mathrm{L}-\left({ }^{3} \mathrm{H}\right)$ fucose. Brain Res. 112: 251-281, 1976.

20. Thompson, S. H. and Getring, P. A. Calcium currents and $\mathrm{K}$-inactivation modulate molluscan soma spikes. Soc. Neurosci. Abstr. 3: 189, 1977. 